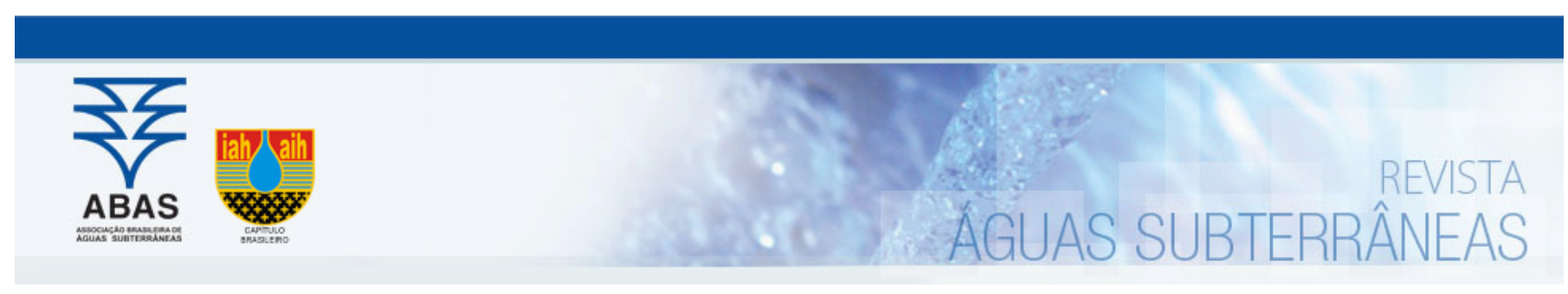

Artigos

\title{
Avaliação física e numérica da formação e quebra de barreira capilar
}

\section{Physical and Numerical Evaluation of Capillary Barrier Formation and Failure}

\author{
Paulo Augusto Diniz Silva ${ }^{1}$; Gilson de F. N. Gitirana Jr. ${ }^{\bowtie}$ \\ 1 Instituto Federal de Educação de Goiás (IFG), Goiânia, GO, Brasil. \\ 2 Universidade Federal de Goiás (UFG), Goiânia, GO, Brasil. \\ $\triangle$ pad.ifg@gmail.com, gilsongitirana@gmail.com
}

\section{Resumo}

Palavras-chave:

Percolação de água.

Barreira capilar.

Ensaio de coluna.

Modelagem numérica.

Método dos volumes finitos.

\begin{abstract}
Este artigo apresenta um estudo do fenômeno de barreira capilar a partir da modelagem física e numérica. Foi desenvolvido um modelo físico unidimensional contendo duas camadas de solos arenosos submetidas a infiltração. A simulação numérica utilizou o método dos volumes finitos para solução da equação de Richards. Os resultados demonstram o efeito e a falha da barreira capilar e sua relação com as propriedades e variáveis de estado do solo. 0 modelo numérico proposto foi capaz de reproduzir adequadamente os fenômenos observados no modelo físico. Demonstrou-se como a barreira capilar depende da diferença de carga piezométrica e condutividade hidráulica não saturada próxima à interface. Foi apresentada a evolução das condições de interface durante o processo de formação e quebra da barreira e demonstrada sua capacidade de recomposição após interrupção da infiltração.
\end{abstract}

Abstract

This paper presents a study of the phenomenon of capillary barrier based on physical and numerical modeling. A one-dimensional physical model was developed comprised of two layers of sandy soils subjected to infiltration. The numerical simulation used the finite volume method to solve Richards' equation. The results show the capillary barrier effect and failure, along with its relationship with the soil properties and state variables. The proposed numerical model was able to adequately reproduce the phenomena observed in the physical model. It has been demonstrated how the capillary barrier depends on the difference of unsaturated hydraulic conductivity and piezometric head between the soil layers next to their interface. Variations in interfacial conditions were presented during the process of capillary barrier formation and failure and its recovery hability after the interruption of infiltration has been demonstrated.

DOI: http://dx.doi.org/10.14295/ras.v31i4.28877

\section{INTRODUÇÃO}

O termo barreira capilar diz respeito ao fenômeno de impedimento do fluxo de água através de camadas estratificadas, que ocorre devido à presença de uma camada de solo sobreposta a outra camada, com textura mais grossa. Essa configuração não é exclusividade dos solos naturalmente estratificados. Barreiras capilares artificiais são empregadas em estruturas de engenharia com o propósito de, por exemplo, melhorar o desempenho de coberturas para o fechamento e proteção de instalações de disposição de resíduos urbanos e industriais.

Apesar da relativa simplicidade descritiva do fenômeno em questão, a modelagem físico-matemática e sua análise não são tarefas triviais. Existem diversas interpretações na literatura sobre como se dá o estabelecimento do efeito de barreira. Tal situação, por vezes, prejudica o estabelecimento de critérios para o dimen- sionamento deste tipo de barreira. De particular interesse é o entendimento de como se dá a dinâmica de manutenção, falha e reestabelecimento do efeito de barreira. Barrès e Bonin (1994) relatam que uma análise detalhada da eficiência dessas barreiras sobre condições naturais é tarefa difícil e muito dispendiosa e que a avaliação da sua eficácia pode levar anos ou até mesmo décadas.

Existem diversas propostas de interpretação do fenômeno de barreira capilar na literatura. Iwata et al. (1995) propõe o balanço das forças verticais em um sistema composto por um capilar de menor diâmetro sobreposto à um capilar de maior diâmetro. Tal análise revela que certa quantidade de água deverá ficar suspensa (retida) sobre a interface, na medida em que a força resultante da tensão capilar da camada superior $\left(\psi_{1}\right)$ seja superior à soma da força resultante da tensão capilar da camada inferior $\left(\psi_{2}\right)$ com o peso da coluna de água acumulada na interface $(h)$. 
Caso a altura da água supere a diferença entre as tensões capilares $\left(h>\psi_{1}-\psi_{2}\right)$ a barreira não mais funcionará como um obstáculo ao fluxo descendente da água.

Billiotte et al. (1988), citado por Oldenburg e Pruess (1993) explicam o fenômeno a partir de outra abordagem, examinando a evolução das condições dos materiais próximos à interface das camadas. Significativas diferenças entre as condutividades hidráulicas próximas à interface resultam em uma restrição ao fluxo de água para a camada do solo grosso. 0 acúmulo de água na camada superior reduz o valor da sucção e eleva sua condutividade hidráulica. Os autores explicam que a falha do efeito de barreira capilar ocorre quando a magnitude da sucção na interface aproxima-se do valor de sucção da entrada de água do solo grosso.

Partindo das hipótese de comportamento de barreiras capilares, diversos autores buscaram avaliar o desempenho desses sistemas na prática. Tem-se, como exemplo, Morris e Stormont (1997), que investigaram o desempenho de dois tipos de barreiras, uma barreira hidráulica de referência e duas barreiras capilares. Estas barreiras foram avaliadas, numericamente, por um período de 10 anos, submetidas às reais condições dos climas de cinco localidades dos EUA. Diante dos resultados, eles concluíram que as barreiras capilares são uma possível alternativa, cujas performances foram iguais ou superiores àquela da barreira hidráulica tomada como referência.

Outro exemplo é o trabalho de Kämpf e Montenegro (1997), que conduziram um estudo experimental com um sistema de cobertura de barreira capilar instalado no aterro sanitário de Am Stempel, Marburg - Alemanha, com dimensões iguais a $15 \mathrm{~m}$ de largura e $40 \mathrm{~m}$ de comprimento. Durante o período de três anos de monitoramento, considerando uma precipitação média anual igual a $650 \mathrm{~mm}$ e uma recarga média anual igual a $300 \mathrm{~mm}$, observou que menos de $20 \mathrm{~mm} /$ ano percolava através da barreira. Com base nesses resultados os autores concluíram que este tipo de barreira é eficiente para impedir a infiltração da água no depósito.

Considerando a importância de barreiras capilares na engenharia, este trabalho busca o entendimento aprofundado do mecanismo de barreira capilar, baseado em experimentação física e análise numérica. 0 principal objetivo do estudo é demonstrar a ocorrência, quebra e recuperação da barreira capilar, procurando interpretar o desenvolvimento do fenômeno com base na observação das variáveis de estado dos solos ao longo do perfil e especialmente na interface entre as camadas, sempre sob a luz das propriedades dos materiais envolvidos e das condições de infiltração impostas na simulações realizadas.

\section{MATERIAIS E MÉTODOS}

Serão descritos nesta seção os materiais e procedimentos experimentais e a abordagem numérica adotada para o estudo.

\subsection{Aparato Experimental}

O aparato experimental foi composto por uma coluna de laboratório, um simulador de chuvas, cinco sensores de umidade e duas balanças (Figura 1). A coluna de laboratório foi desenvolvida com dois segmentos de tubos de acrílico de $1,5 \mathrm{~cm}$ de espessura, com $10 \mathrm{~cm}$ de diâmetro interno e $50 \mathrm{~cm}$ de altura para cada segmento. O sistema é dotado de uma base com registros de entrada e saída de água e ar e de orifícios para a instalação dos sensores de umidade, a $5,20,45,60$ e $80 \mathrm{~cm}$, a partir do topo da coluna.

O simulador de chuvas é composto por dois reservatórios de água, sendo um destinado a alimentar e manter a carga hidráulica constante no sistema e outro contendo um conjunto de nove agulhas hipodérmicas de aço inox, com $6 \mathrm{~mm}$ de comprimento e $0,7 \mathrm{~mm}$ de diâmetro. 0 simulador de chuva permite a distribuição uniforme de água no topo da coluna de solo. Este aparelho é capaz de produzir diferentes taxas de precipitação, conforme a quantidade de agulhas e a altura de água empregada no reservatório responsável pela distribuição da água.

O monitoramento das variações temporais e espaciais do conteúdo de água ocorridas na coluna foi feita através de cinco sensores de umidade do tipo capacitivo, modelo EC-5, fabricado pela empresa Decagon Devices. Este modelo possui faixa de medição de 0 a 100\% da umidade volumétrica saturada, um volume de influência de $240 \mathrm{~mL}$, e acurácia de $\pm 3 \%$ quando é utilizada a calibração de fábrica. Todos os sensores foram devidamente calibrados para os materiais utilizados, de maneira que foi obtida acuraria de $1 \%$. Os dados dos sensores são simultaneamente obtidos utilizando um coletor de dados programável.

O balanço hídrico é feito por duas balanças de precisão. A primeira balança, com capacidade de $30 \mathrm{~kg}$ e resolução e reprodutibilidade de $5 \mathrm{~g}$, registra a massa total da coluna. A segunda balança, com capacidade de $3 \mathrm{~kg}$ e resolução e reprodutibilidade de $1 \mathrm{~g}$, registra, exclusivamente, a massa de água drenada. 
Figura 1 - Painel fotográfico do aparato experimental

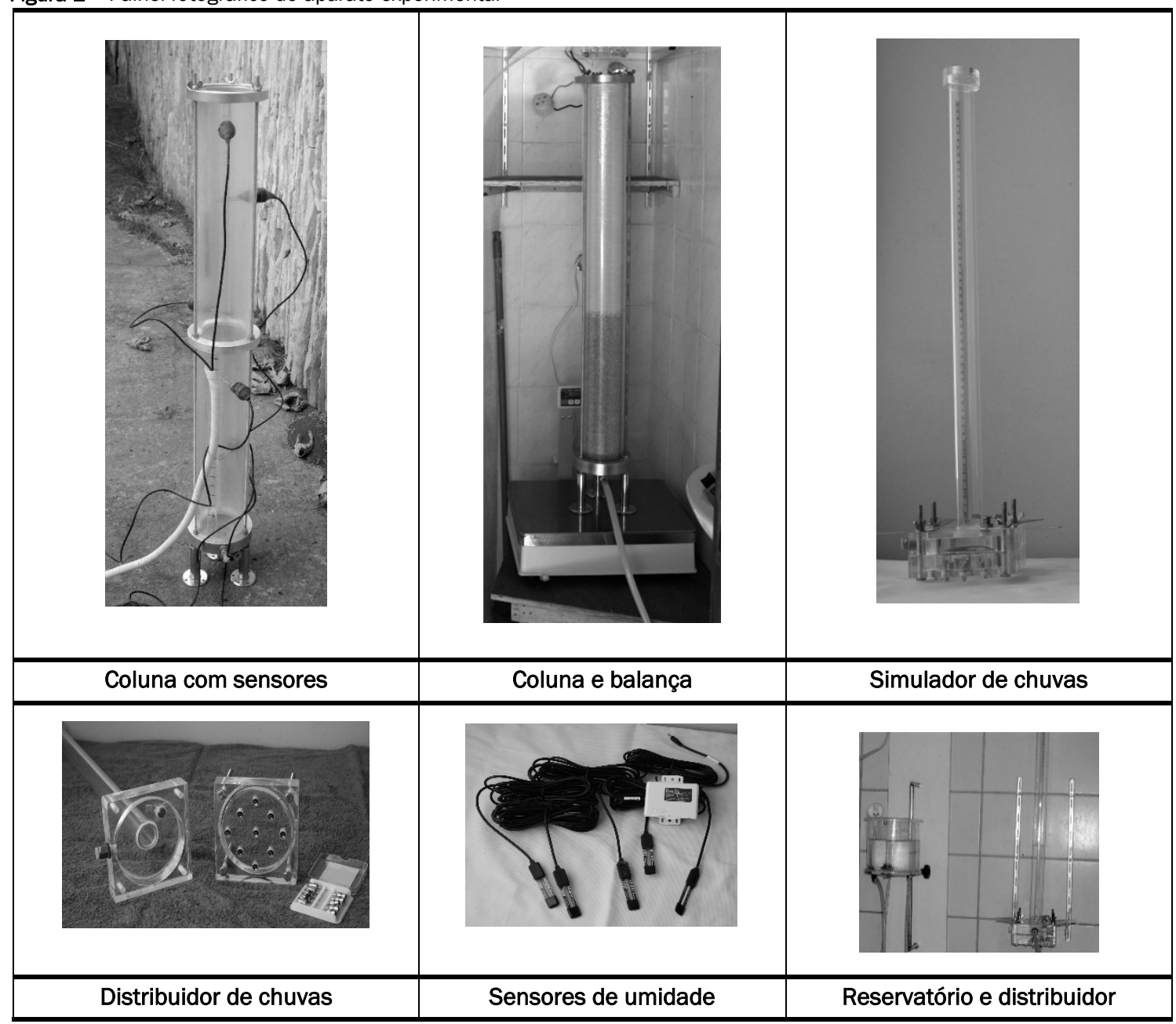

\subsection{Materiais utilizados, moldagem da coluna e simulação da barreira capilar}

Para compor a coluna de laboratório foram utilizados dois tipos de areias. As areias são classificada pela ABNT (1982) como sendo de granulometria fina e grossa e são denominadas como IPT 100 e IPT 16, respectivamente. Os parâmetros dos experimentos e as propriedades dos materiais são apresentadas na Tabela 1. Os materiais foram compactados de maneira controlada, de forma a serem obtidos valores de compacidade relativa e porosidade especificados. Os valores foram especificados com base em critérios como baixa deformabilidade final e facilidade de reprodução ao longo do programa experimental.

Os sensores capacitivos foram instalados durante o processo de compactação, sendo posicionados com orientação horizontal. A base da coluna recebeu uma camada de pedrisco com $10 \mathrm{~cm}$ de espessura, para evitar a ascensão capilar na base do sistema, bem como para auxiliar a drenagem. Considerou-se, para fins práticos, que a camada de pedrisco não participou da dinâmica do fluxo de água.

O cenário proposto consiste na simulação física da infiltração da água, sob ação de drenagem gravitacional, durante um período de 10 horas, com períodos alternados de precipitação e estiagem, tal como detalhado na Tabela 1. Tratam-se de três eventos de precipitação. 0 primeiro evento teve duas horas de duração e intensidade constante de $9,1 \pm 0,5 \mathrm{~cm} / \mathrm{h}$. Os dois eventos subsequentes tiverem intensidade de $9,3 \pm 0,5 \mathrm{~cm} / \mathrm{h}$ e uma hora de duração. Após cada um dos eventos de precipitação foi realizado um evento, denominado de 'redistribuição', no qual não houve precipitação e foi observado o avanço da frente de molhagem e o comportamento da barreira. Esta sequencia de eventos de precipitação e redistribuição foi estabelecida de manteira a provocar a formação, quebra e reestabelecimento da barreira capilar. 
Tabela 1 - Características de compactação do solo, eventos simulados e parâmetros hidráulicos

\begin{tabular}{|c|c|c|c|c|c|c|}
\hline \multicolumn{7}{|c|}{ Características de moldagem da coluna de solo } \\
\hline \multicolumn{3}{|l|}{ Material } & \multicolumn{2}{|l|}{ Areia IPT 100} & \multicolumn{2}{|c|}{ Areia IPT 16} \\
\hline \multicolumn{3}{|l|}{ Altura $(\mathrm{cm})$} & \multicolumn{2}{|l|}{50} & \multicolumn{2}{|c|}{40} \\
\hline \multicolumn{3}{|c|}{ Conteúdo de água inicial $\left(\mathrm{cm}^{3} / \mathrm{cm}^{3}\right)$} & \multicolumn{2}{|l|}{$0,070 \pm 0,005$} & \multicolumn{2}{|c|}{$0,015 \pm 0,005$} \\
\hline \multicolumn{3}{|c|}{ Massa específica úmida $\left(\mathrm{g} / \mathrm{cm}^{3}\right)$} & \multicolumn{2}{|l|}{$1,598 \pm 0,02$} & \multicolumn{2}{|c|}{$1,527 \pm 0,03$} \\
\hline Compacidade re & & & \multicolumn{2}{|l|}{$0,95 \pm 0,03$} & \multicolumn{2}{|c|}{$0,95 \pm 0,03$} \\
\hline \multicolumn{7}{|c|}{ Características dos eventos } \\
\hline Eventos & $\begin{array}{l}\text { Duração } \\
\text { (h) }\end{array}$ & $\begin{array}{l}\text { Período } \\
\text { (h) }\end{array}$ & $\begin{array}{l}\text { Massa de água } \\
\text { média (g) }\end{array}$ & $\begin{array}{c}\text { Vazão média } \\
\left(\mathrm{cm}^{3} / \mathrm{h}\right)\end{array}$ & \multicolumn{2}{|c|}{$\begin{array}{c}\text { Taxa de precipitação } \\
(\mathrm{cm} / \mathrm{h})\end{array}$} \\
\hline Precipitação & 2 & 0 às 2 & $1430 \pm 10$ & $712 \pm 5$ & \multicolumn{2}{|r|}{$9,1 \pm 0,5$} \\
\hline Redistribuição & 2 & 2 às 4 & $650 \pm 5$ & - & \multicolumn{2}{|r|}{-} \\
\hline Precipitação & 1 & 4 às 5 & $717 \pm 10$ & $717 \pm 5$ & \multicolumn{2}{|r|}{$9,1 \pm 0,5$} \\
\hline Redistribuição & 2 & 5 às 7 & $440 \pm 5$ & - & \multicolumn{2}{|r|}{-} \\
\hline Precipitação & 1 & 7 às 8 & $732 \pm 10$ & $732 \pm 5$ & \multicolumn{2}{|r|}{$9,3 \pm 0,5$} \\
\hline Redistribuição & 2 & 8 às 10 & $452 \pm 5$ & - & \multicolumn{2}{|r|}{-} \\
\hline \multicolumn{7}{|c|}{ Parâmetros hidráulicos de van Genuchten (1980) } \\
\hline Material & $K_{s}(\mathrm{~cm} / \mathrm{s})$ & $\alpha\left(\mathrm{cm}^{-1}\right)$ & $n$ & $m$ & $\theta_{\mathrm{r}}$ & $\theta_{\mathrm{s}}$ \\
\hline Areia IPT 100 & $1,215 \times 10^{-2}$ & 0,02696 & 6,1448 & 0,8372 & 0,037 & 0,402 \\
\hline Areia IPT 16 & $5,97 \times 10^{-1}$ & 0,1393 & 4,1680 & 0,7590 & 0,010 & 0,415 \\
\hline
\end{tabular}

\subsection{Modelo Matemático}

A interpretação do processo de formação, quebra e restituição do fenômeno de barreira capilar pode ser facilitada ao unir as observações experimentais e a avaliação matemática do fenômeno. A equação de Richards, aqui empregada, é resultante da combinação da equação de conservação de massa em um elemento infinitesimal representativo, da Lei de Darcy-Buckinghan para a taxa de fluxo de água no solo saturado/não saturado e das características de armazenamento de água do material. A equação pode ser expressa como (HILLEL, 1998):

$$
C(\psi) \frac{\partial h}{\partial t}=\frac{\partial K(\psi)}{\partial z} \frac{\partial h}{\partial z}+K(\psi) \frac{\partial^{2} h}{\partial z^{2}}
$$

onde $C(\Psi)$ é a função de capacidade de armazenamento de água [ $\left.\mathrm{L}^{-1}\right]$, definida como a derivada da curva de retenção, ou seja, a variação de umidade volumétrica dividida pela variação correspondente de carga piezométrica; $\psi$ é a sucção mátrica [L]; $h$ é a carga hidráulica [L]; $h=z+h_{p} ; h_{p}$ é a carga piezométrica, $h_{p}=$ $u_{w} / \gamma_{w} ; u_{w}$ é a poropressão de água [ML-1T-2]; $\gamma_{w}$ é o peso específico da água [ML-2T-2]; $K(\psi)$ a condutividade hidráulica na condição não saturada [LT-1] e $z$ a elevação [L].

Para a definição da capacidade de armazenamento de água foi empregada a relação de van Genuchten (1980), dada por:

$$
S_{e}=\frac{\theta-\theta_{r}}{\theta_{s}-\theta_{r}}=\frac{1}{\left[1+(\alpha \psi)^{n}\right]^{m}}
$$

onde $S_{e}$ é a saturação efetiva [-]; $\theta_{r}$ é o conteúdo volumétrico de água residual [ $\left.\mathrm{L}^{3} \mathrm{~L}^{-3}\right] ; \theta_{\mathrm{s}}$ é o conteúdo volumétrico de água na condição saturada [ $\mathrm{L}^{3} \mathrm{~L}^{-3}$ ]; $\alpha$ é uma constante de ajuste relativa ao valor da sucção na entrada de ar no solo [ $\left.\mathrm{L}^{-1}\right]$; $n$ e $m$ são constantes de ajuste que representam a distribuição do tamanho dos poros [-].

Para determinação da permeabilidade dos materiais na condição não saturada foi utilizado o modelo de Mualem (1976), juntamente com a Equação 2:

$$
K(\psi)=K_{s} S_{e}^{0,5}\left[1-\left(1-S_{e}^{1 / m}\right)^{m}\right]^{2}
$$

onde $K(\psi)$ e $K_{s}$ são a condutividade hidráulica na condição não saturada e saturada, respectivamente [LT-1] e $m=1-1 / n$.

Os parâmetros hidráulicos das Equações 2 e 3 podem ser encontrados na Tabela 1. É importante destacar que tais parâmetros foram determinados experimentalmente utilizando um Funil de Haines e em trajetórias de secagem. Foram sempre empregadas amostras compactadas com a mesma porosidade adotada para os ensaios de coluna. Desta maneira, os parâmetros hidráulicos são representativos dos materiais nos ensaios de coluna, salvo eventuais efeitos de histerese que não foram objeto desta pequisa. 


\subsection{Solução Numérica e Simulação do Cenário}

A solução numérica da Equação 1 foi baseada no método dos volumes finitos, utilizando o esquema de diferenças centrais para a derivada espacial e um esquema implícito para a derivada temporal. A discretização do domínio resulta em um sistema linear de equações algébricas formando uma matriz tri-diagonal que é resolvida iterativamente pelo algoritmo de Thomas - Tridiagonal Matrix Algorithm.

O esquema numérico e estratégias de solução adotados foram descritos em detalhe por Silva et al. (2010). A condutividade hidráulica nas interfaces do volume de controle foi avaliada a partir da média geométrica, tal como proposto por Haverkamp e Vauclin (1979) e Yeh e Harvey (1990). O critério de convergência adotado foi igual a $10^{-6} \mathrm{~cm}$ entre os valores relativos de carga hidráulica. Foi realizado também o balanço de massa instantâneo durante todo o evento simulado, tendo sido verificado um erro máximo de aproximadamente 0,25\%.

O cenário simulado numericamente é idêntico àquele modelado fisicamente, contendo duas camadas horizontais sobrepostas, areia IPT 100 sobre a areia IPT 16, com espessuras iguais a 50 e $40 \mathrm{~cm}$, respectivamente. 0 sistema é mantido sob ação de drenagem gravitacional, durante um período de 10 horas.

A modelagem numérica utilizou uma malha igualmente espaçada de $0,1 \mathrm{~cm}$ e um incremento de tempo igual a 1 segundo. $A$ condição inicial utilizada para todo o domínio foi especificada como um valor uniforme de carga piezométrica correspondente à umidade volumétrica de moldagem. A camada superior (areia IPT 100) possui como condições iniciais $S_{e}=10 \%$ e $h_{p}=-60$ $\mathrm{cm}$. Já a camada inferior (areia IPT 16) possui $S_{e}=2 \%$ e $h_{p}=-$ $24 \mathrm{~cm}$.

Para as condições de contorno, foi empregada no topo uma condição de fluxo com taxa correspondente à precipitação aplicada no modelo físico. Conforme apresentado na Tabela 1, tratam-se de períodos intercalados de precipitação e interrupção de fluxo na superfície, estabelecidos de maneira a demonstrar a formação, quebra e a reconstituição do efeito de barreira capilar.

Para a base da coluna foi adotada a condição de livre drenagem. Tal condição é implementada utilizando uma função condicional:

$$
\left\{\begin{array}{l}
\text { se }: h_{p} \leq 0 \text { então } q=0 \\
\text { se }: h_{p}>0 \text { então } q=K_{s}
\end{array}\right.
$$

onde $q\left[\mathrm{LT}^{-1}\right]$ é a taxa de fluxo aplicada na fronteira. Tem-se, desta maneira, que o fluxo é nulo quando a carga piezométrica é negativa e que uma taxa de fluxo negativa (i.e., de saída) igual à permeabilidade saturada é aplicada quando a carga piezométrica é positiva. Esta segunda condição é matematicamente equivalente à especificar a carga piezométrica na fronteira como sendo nula.

\section{RESULTADOS E DISCUSSÃO}

A simulação do cenário é apresentada, primeiramente, avaliando o período inicial de 0 a 4 horas, no qual são detalhados o efeito e a falha da barreira capilar. Posteriormente, é examinado o período entre 4 e 10 horas, em que ocorre a recuperação da barreira.

\subsection{Formação do Efeito de Barreira Capilar e Posterior Falha}

Definiu-se, neste trabalho, que o tempo transcorrido durante a acumulação da água sobre a interface, sem qualquer drenagem da água na camada inferior, corresponde à manifestação do efeito de barreira capilar. A duração deste período é de difícil previsão, sendo condicionada pela taxa de precipitação induzida ao sistema, pela condutividade hidráulica dos meios e pelo conteúdo de água presente na condição inicial, dentre outros fatores. A falha da barreira é caracterizada pela drenagem de água pela camada inferior em resposta a qualquer quantidade de água acrescida à interface entre os materiais. Neste momento, tem-se uma condição limite de equilíbrio das forças capilares e gravitacional.

A evolução temporal do volume de água infiltrado, armazenado e drenado observado no modelo físico é mostrada na Figura 2. A verificação do balanço de massa revelou erros sempre inferiores a $1 \%$. Valores temporais e espaciais do conteúdo de água são apresentados na Figura 3. A quebra da barreira capilar se dá em algum momento ligeiramente anterior ao início do registro de drenagem na base do sistema. A partir da ruptura da barreira, observa-se constância na quantidade de água armazenada na coluna. Ou seja, a ruptura da barreira resulta na incapacidade de armazenamento de quantidades adicionais de água precipitada, mas não prejudica aquela quantidade já armazenada, que permanece no solo. Ocorreram mudanças significativas nos conteúdos de água próximo da interface entre as camadas de areia, principalmente no intervalo entre 55 a 105 minutos de simulação. 0 conteúdo volumétrico de água na camada superior variou neste intervalo de tempo de 0,17 a $0,40 \mathrm{~cm}^{3} / \mathrm{cm}^{3}$. Já a camada inferior sofreu uma variação de 0,02 a $0,11 \mathrm{~cm}^{3} / \mathrm{cm}^{3}$, aproximadamente.

Verifica-se que a frente de molhamento alcança a interface entre as duas camadas de areia, aproximadamente aos 55 minutos do início da infiltração. A frente torna-se mais pronunciada, na medida em que a água vai se acumulando sobre a interface. Decorridos 75 minutos, quando o conteúdo de água da areia IPT 100 se aproxima da condição saturada, ocorre uma mudança significativa no conteúdo de água na profundidade de $60 \mathrm{~cm}$ (areia IPT 16). Neste momento, pode-se afirmar que uma quantidade de água atravessou a interface da barreira capilar e ela deixou de funcionar como um anteparo hidráulico. Neste caso, a restrição à passagem da água durou pelo menos 20 minutos - diferença entre os momentos 55 e 75 minutos. Decorridos 105 minutos, dá-se início à drenagem de água na base. 
Figura 2 - Evolução temporal do volume de água no modelo físico

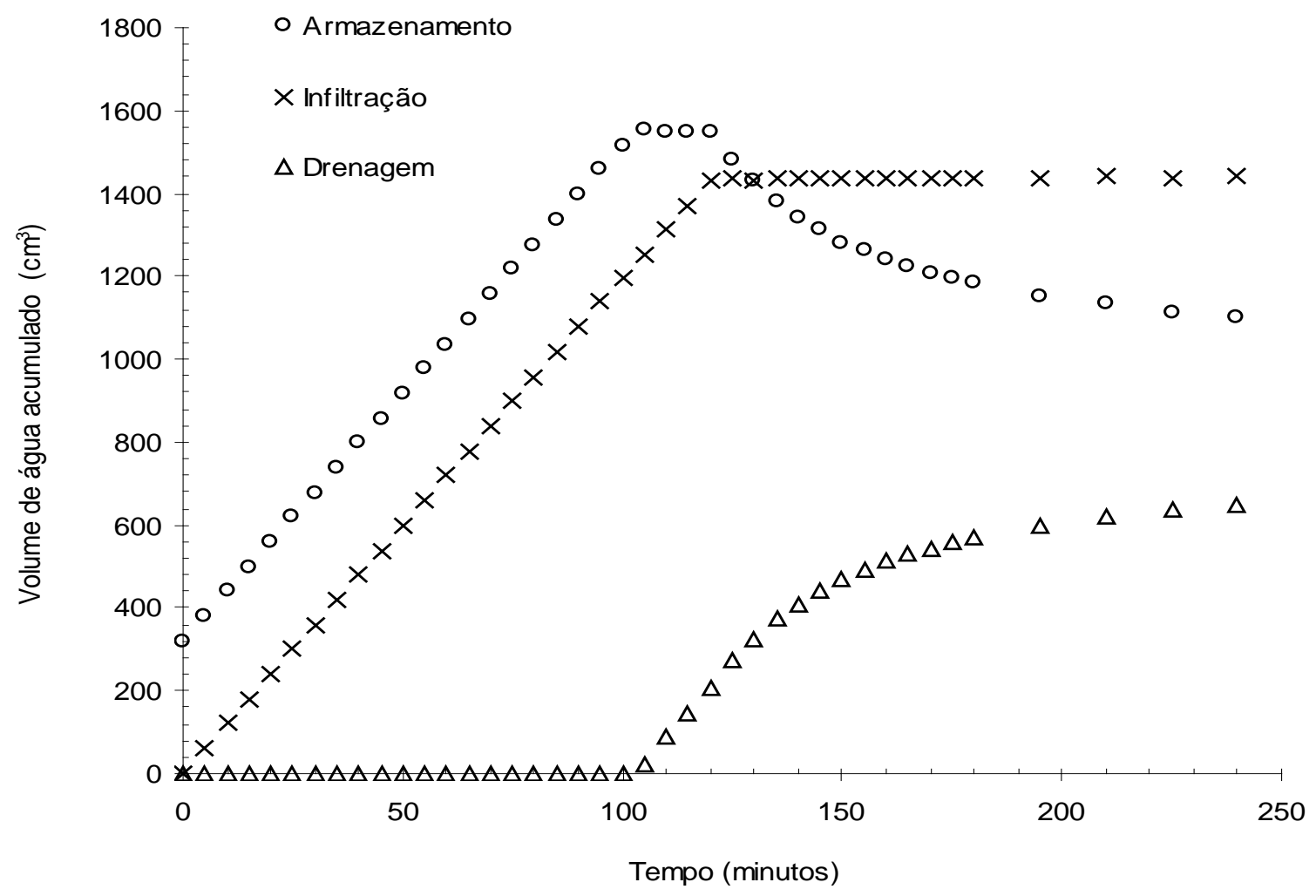

Figura 3 - Perfil de conteúdo volumétrico de água observado no modelo físico durante a infiltração Conteúdo de água $\left(\mathrm{cm}^{3} / \mathrm{cm}^{3}\right)$

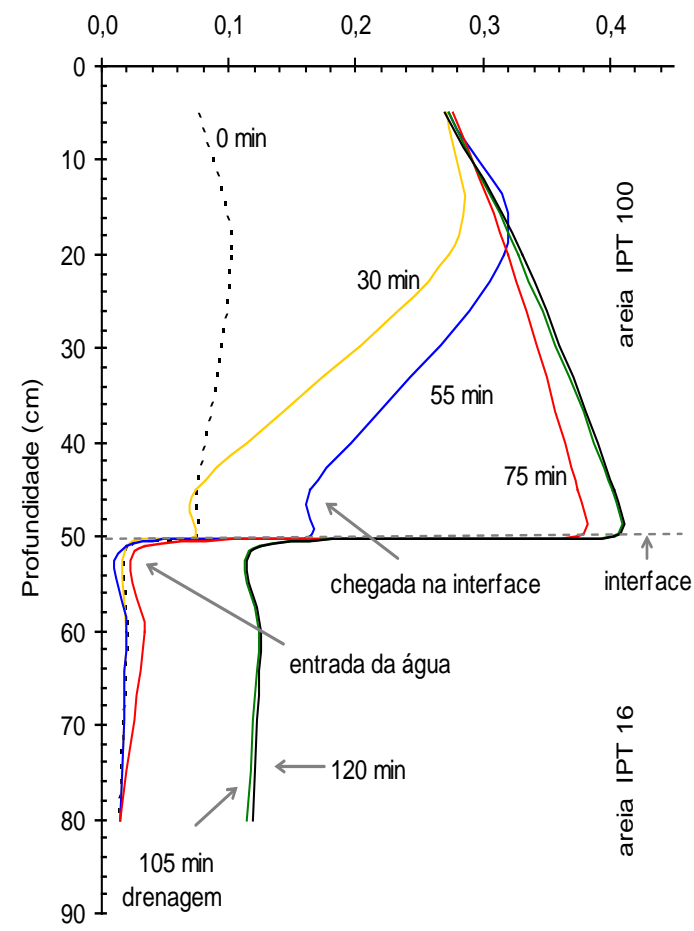


A comparação dos perfis de conteúdo volumétrico de água observados no modelo físico e obtidos pela simulação numérica é apresentada na Figura 4. Os resultados mostram que o modelo numérico reproduziu as tendências gerais do deslocamento da água no solo, indicadas pelos dados experimentais. As diferenças mais significativas entre os dados do modelo físico e a simulação numérica ocorreram na camada inferior, com diferenças relativas médias de $14,5 \%$. Já na camada superior, o erro relativo médio foi de apenas $5,6 \%$.

Na Figura 4a, período marcado pela infiltração, destacam-se os seguintes eventos: (i) passados 60 minutos, a chegada e início do acúmulo da água na interface; (ii) passados 75 minutos, a entrada da água na camada inferior e (iii) passados 105 minutos, a iminência da chegada da água na base da coluna.

Já na Figura 4b, período caracterizado pela falha da barreira capilar e redistribuição do conteúdo de água, o padrão do fluxo da camada superior é, essencialmente, influenciado pela presença da lâmina de água sobre a interface interna. Por sua vez, na camada inferior, livre dessa circunstância, a frente de molhamento corresponde à de drenagem gravitacional, com distribuição uniforme de umidade volumétrica. A dinâmica da água exibida na experimentação física e numérica seguiu o mesmo padrão de comportamento apresentado na literatura (ZASLAVSKY, 1964; MIYAZAKI, 1993; HILLEL, 1998, dentre outros).

Figura 4 - Perfis de conteúdo volumétrico de água desenvolvidos no modelo físico e na simulação numérica durante: a) infiltração e drenagem; b) redistribuição
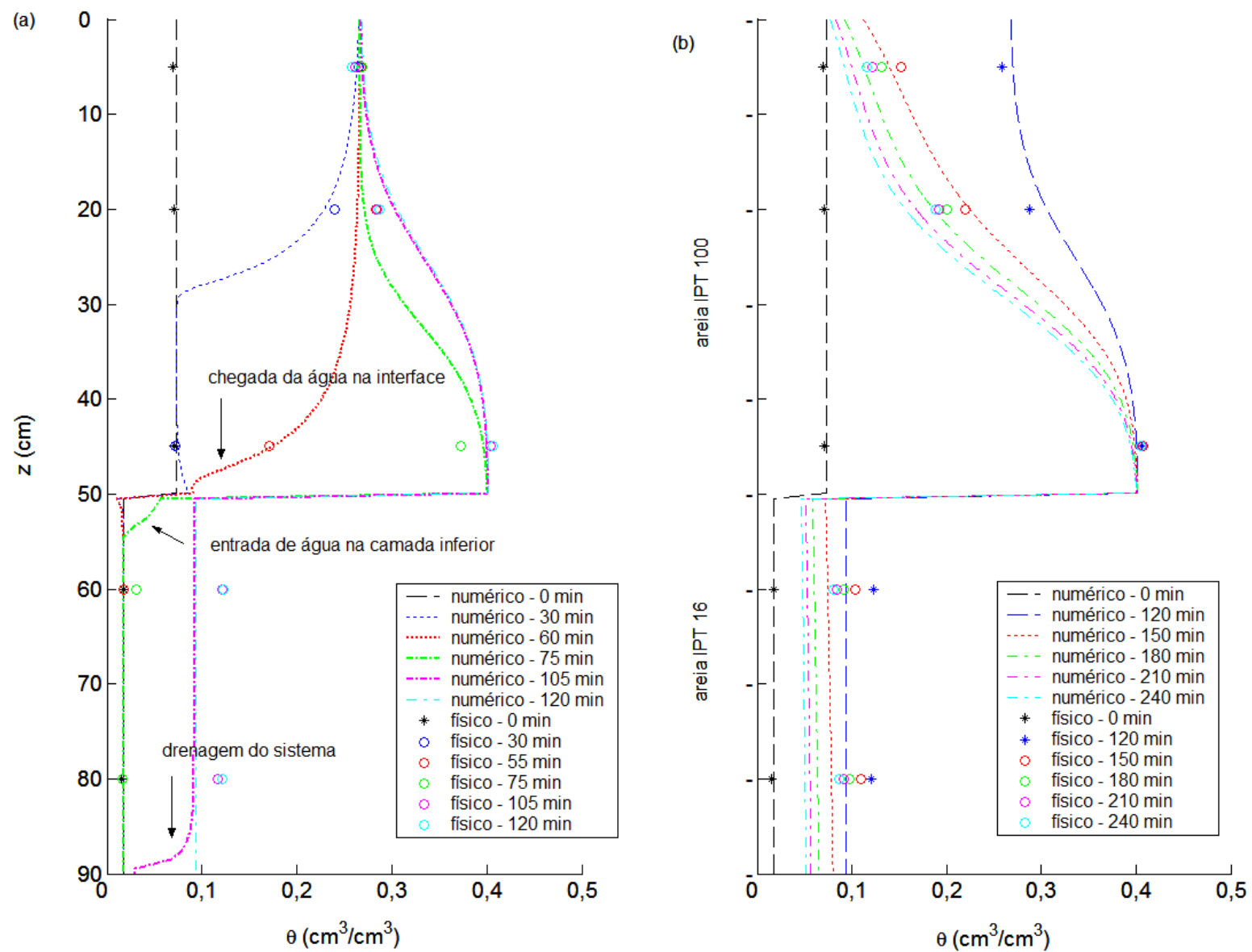
As variações de condutividade hidráulica, grau de saturação efetivo e carga piezométrica ocorridas nas proximidades da interface da barreira durante a simulação do fenômeno pode ser observada na Figura 5 . As variáveis são calculadas imediatamente acima e abaixo da interface, a uma distância igual a metade do tamanho do volume de controle adotado na discretização do domínio, $\Delta z / 2$. Desta maneira, a distância entre os pontos monitorados foi de $0,1 \mathrm{~cm}$. Na Figura 5 a pode-se observar que a entrada de água na camada inferior ocorre quando o grau de saturação da camada superior se aproxima de $1 \mathrm{e}$, por consequência a sua condutividade hidráulica aproxima-se da condutividade hidráulica saturada e quando os valores das condutividades hidráulicas dos materiais das duas camadas apresentam a menor diferença entre eles.

Na Figura $5 b$ destacam-se os três estágios previamente observados com o auxílio da Figura 4a. As etapas de surgimento e duração do efeito de barreira capilar e de falha da barreira possuem importante relação com as condições de permeabilidade e saturação na interface. Pode-se destacar a marcante elevação da permeabilidade da face inferior da interface que desencadeia, eventualmente, o rompimento de barreira. Por outro lado, a acumulação de água, retratada pela elevação de grau de saturação efetivo, também pode ser relacionada com a fase de manutenção da barreira.

Pode também ser feita a tentativa de relação entre o momento de quebra da barreira capilar e as cargas piezométricas próximas à interface, apresentadas na Figura $5 \mathrm{c}$. Considerando pontos muito próximos da interface, a diferença de carga gravimétrica se torna irrelevante e o potencial produtor de fluxo é proveninente de gradientes de carga piezométrica. Enquanto o material superior possuir carga piezométrica inferior à do material inferior, não seria possível fluxo descentente. Os resultados obtidos indicam que até a chegada da água na interface, o valor da carga em cada substrato se mantem próximo aquele estipulado para representar a condição inicial. A partir desse ponto e, na medida em que, a água vai-se acumulando na interface, a camada superior experimenta elevação na sua carga piezométrica, chegando a superar o valor da carga da camada inferior. Quando as cargas piezométricas das duas areias se igualam novamente ocorre a falha da barreira. 0 restante do processo se dá com uma diferença mínima entre as cargas dos materiais envolvidos.

Figura 5 - Evolução das condições na interface entre os materiais obtida numericamente: a) K; b) $S_{e}$; c) $h_{p}$

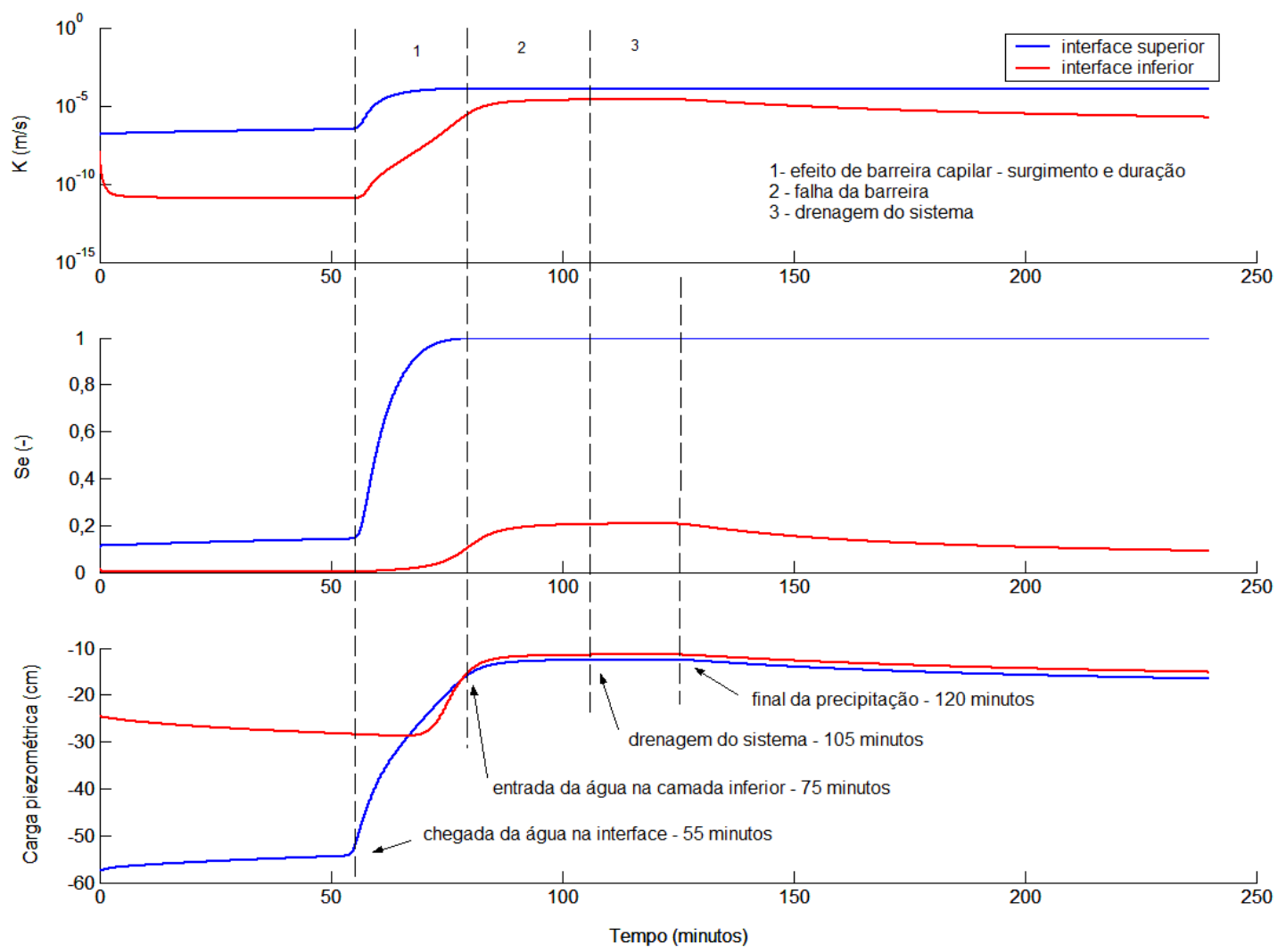

A Fig. $5 c$ revela também que a quebra da barreira parece corresponder à um valor limite de carga piezométrica, atingido após o acúmulo de água sobre a interface. A evolução dos valores de carga piezométrica corresponde às variações nos valores de grau de saturação efetivo e de permeabilidade que permitem a interpretação do mecanismo de quebra da barreira, conforme explicado anteriormente. A comparação entre cargas piezométricas e tensões capilares limite, sugerida por Iwata et al. (1995), pode também auxiliar na interpretação do mecanismo de quebra da barreira. No caso do materiais em questão, tem-se uma diferença entre valores de tensão capilar determinadas pelo parâmetro $\alpha$ (Tabela 1 ) de aproximadamente $12 \mathrm{~cm}$. Por outro lado, a Figura 4 apresenta acumulação de pouco menos de $10 \mathrm{~cm}$ água logo após o momento de ruptura da coluna (i.e., tempo de 75 minutos). A relação proposta por Iwata et al. (1995) parece se confirmar, apesar dos valores não serem idênticos aos esperados. 
Finalmente, no último estágio, de drenagem na base da coluna, o volume da água que atravessa a barreira vai, aos poucos, sendo percolado para camadas mais profundas devido à ação da gravidade. Com a passagem do tempo, a camada inferior volta a funcionar como anteparo ao fluxo de água imposto pela camada superior, na medida em que o volume de água presente na camada for sendo drenado.

\subsection{Recuperação da Barreira Capilar}

A recuperação da barreira capilar no modelo físico é identificada pela capacidade do sistema em acumular água sem que ocorra uma drenagem significativa, tal como mostrado na Figura 6 , destacado pelo segundo e terceiro ciclo. Considera-se, neste trabaIho, que a recuperação do efeito de barreira capilar somente ocorrerá quando o sistema deixar o estado de equilíbrio limite. Isto quer dizer que, a altura da água acumulada na interface de- verá ser menor do que a da composição das forças capilares ( $h$ $<\psi_{1}-\psi_{2}$ ), para que o sistema readmita um acréscimo de água e volte a funcionar como anteparo ao fluxo da água. 0 tempo de recuperação depende da redistribuição da água ou da drenagem interna no perfil.

O resultado da simulação computacional pode ser novamente apreciado a partir da evolução temporal do volume de água ocorrido na experimentação, da forma e posição da frente de molhamento no interior do perfil de solo e do balanço de massa. $\mathrm{Na}$ Figura 6 pode-se perceber que ocorreu uma boa aproximação entre os volumes de água estimados pelo modelo computacional e aqueles observados pelo modelo físico. Foi observado um erro máximo de 7,0\% nos valores de volume armazenado, sendo tais erros máximos concentrados próximos do fim de cada evento de precipitação

Figura 6 - Recuperação da barreira capilar observada no modelo físico

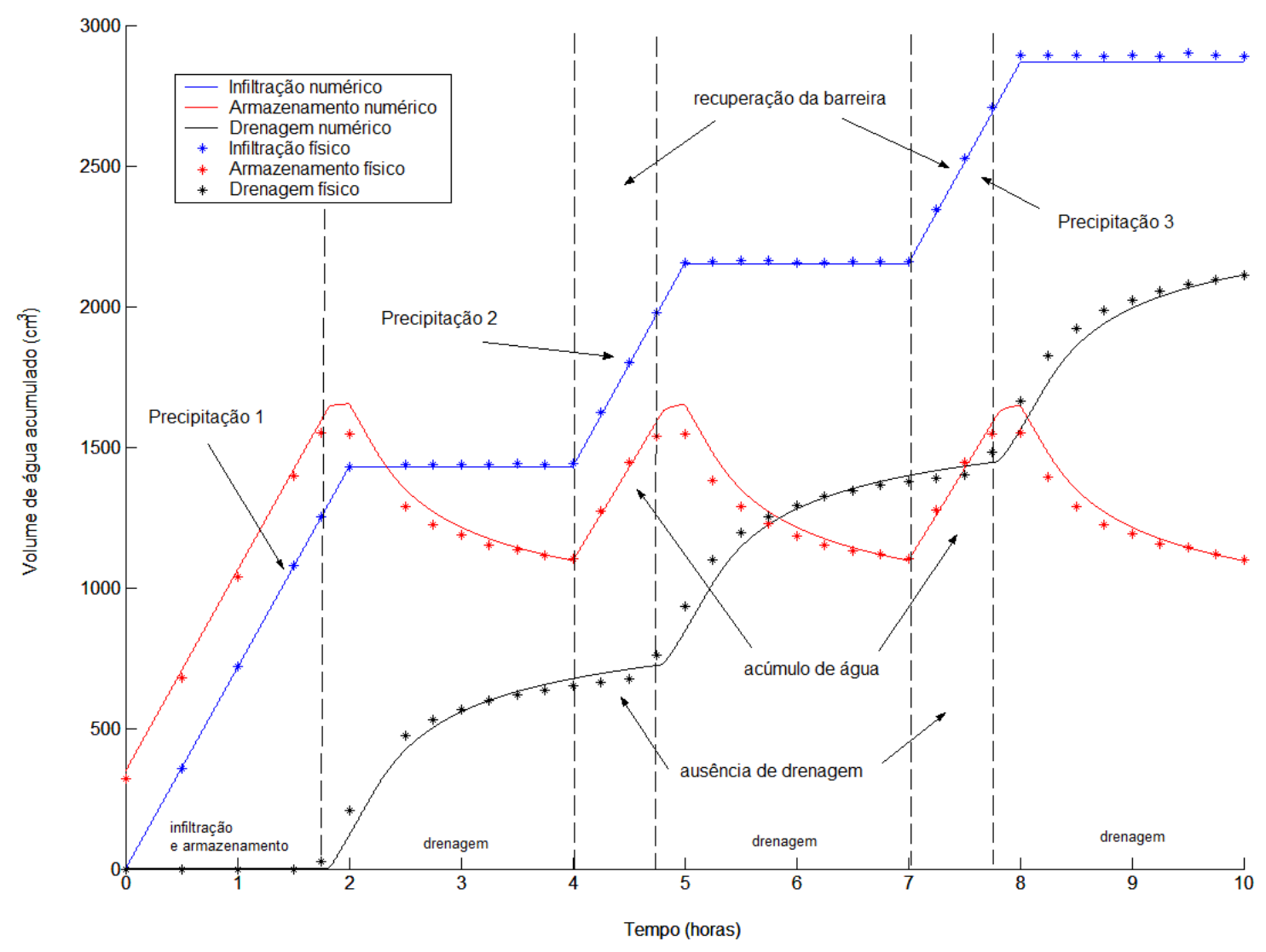

A Figura 7 apresenta o comportamento hidráulico da interface entre as duas areias, demonstrando que a recuperação da barreira capilar ocorre quando a diferença entre as condutividades hidráulicas dos meios envolvidos é de quase duas ordens de magnitude. Nesta situação, o sistema admite um novo acréscimo de água e a interface volta a atuar como barreira hidráulica ao fluxo de água.

A Figura 8 apresenta a dinâmica da água na experimentação física e numérica, com mecanismos de retenção e drenagem que obedecem a composição das forças de campo, resultante das forças capilares e gravitacional. As frentes de molhamento são bastante semelhantes durante todo período do experimento. $\mathrm{Na}$ camada superior o formato é característico de um solo uniforme, com gradual elevação do conteúdo de água com a profundidade até o acúmulo de água proporcionado pela barreira capilar, atingindo o conteúdo volumétrico de água correspondente à saturação. Na camada inferior a distribuição de umidade é própria daquela estabelecida pela drenagem gravitacional, com valores constantes ao longo da profundidade, devido à elevada permeabilidade do material do estrato em questão e à livre drenagem no limite inferior. 
Figura 7 - Diferença de $\mathrm{K}$ na interface entre as duas camadas obtida via simulação numérica

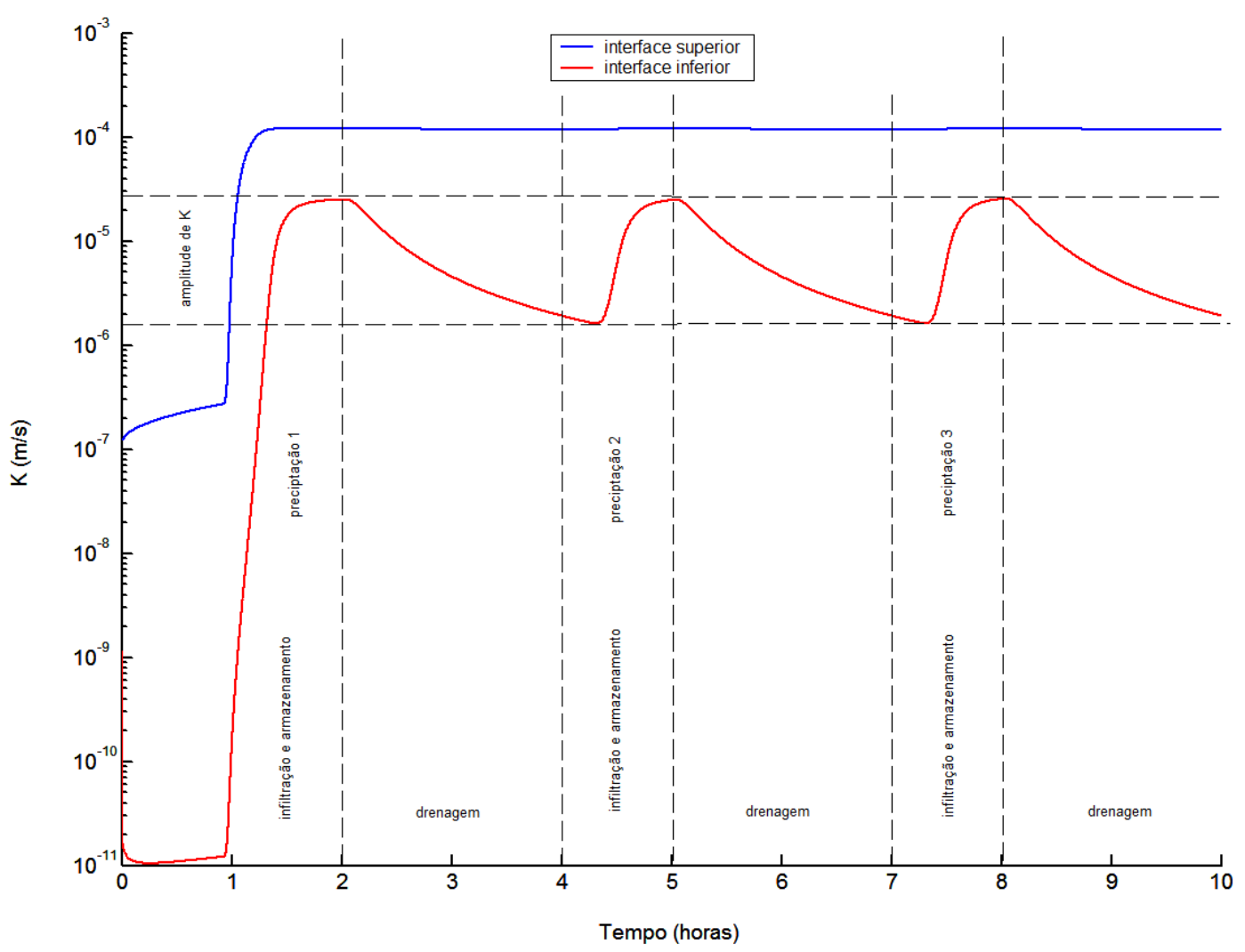

Figura 8 - Evolução da frente de molhamento obtida utilizando os modelos físico e numérico
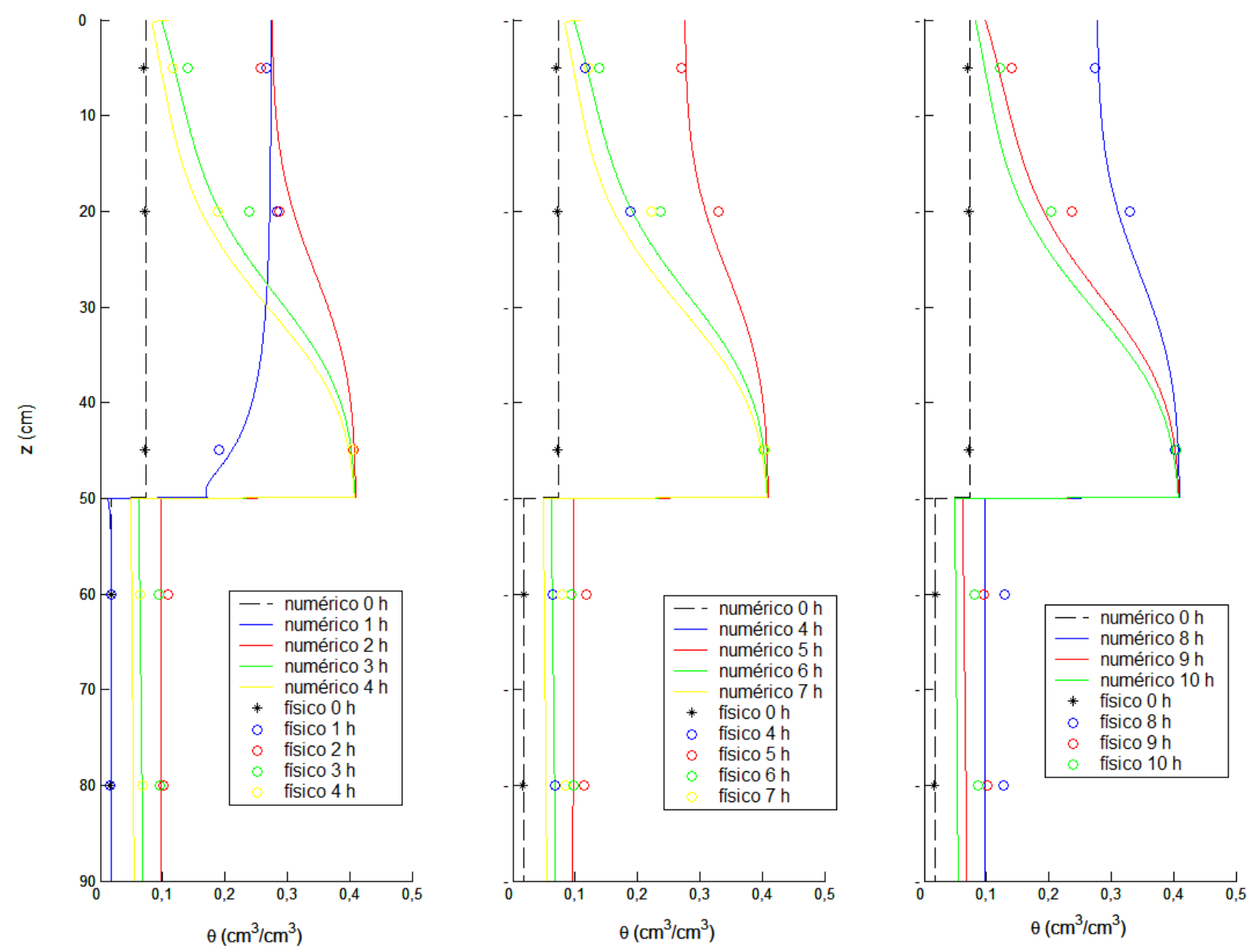


\section{CONSIDERAÇÕES FINAIS}

O presente trabalho apresentou a simulação física e numérica do fenômemo de barreira capilar, mostrando como ele ocorre, desde seu surgimento, passando pela sua falha, até a sua recuperação.

Os resultados mostram que o fenômeno pode ser relacionado à diferença de condutividade hidráulica entre os meios envolvidos. 0 efeito de barreira capilar foi proporcionado pela significativa diferença entre as condutividades próximas da interface. Na medida em que essa diferença foi reduzida pela chegada da água na camada superior, ocorreu a falha da barreira. A recuperação da barreira, após a interrupção do fornecimento de água ao sistema, se deu quando a diferença entre as condutividades das duas camadas de areias tornou a se elevar, atingindo em torno de duas ordens de grandeza. A partir deste momento, o meio tornou-se capaz de aceitar um novo acúmulo de água sobre a interface.

A formação da barreira capilar foi relacionada também com o desenvolvimento de cargas piezométricas próximas à interface e com a formação de uma coluna de material saturado acima da interface.

Com respeito à comparação entre as simulações física e numérica, foram observadas ligeiras diferenças entre elas, que não prejudicaram a interpretação do fenômeno. Foi apresentada no trabalho a hipótese de que tais discrepâncias podem ser devidas à não consideração da histerese no modelo numérico.

Espera-se que o presente estudo possa auxiliar na interpretação das variáveis e mecanismos de fluxo presentes nos projetos utilizando esse tipo de barreira. Barreiras capilares podem ser utilizadas em associação ou em alguns casos substituir as tradicionais barreiras hidráulicas, permitindo o emprego de materiais alternativos e oferecendo diferentes alternativas. Além disso, a adoção desse tipo de barreira reduz parcialmente a dependência de materiais argilosos com baixo valor de condutividade hidráulica em sistemas que exigem estanqueidade.

\section{REFERÊNCIAS}

ASSOCIAÇÃO BRASILEIRA DE NORMAS TÉCNICA. NBR 7214 - Agregados - Areia Normal para Ensaio de Cimento. Rio de Janeiro: ABNT, 1982.
BARRĖS, M.; BONIN, H. The Capillary Barrier for Surface Capping. CHRISTENSEN, T.H.;. COSSU, R.; STEGMANN, R. (ED.). Landfilling of waste: Barriers, 139-148. 1994

HAVERKAMP, R.; VAUCLIN, M. A Note on Estimating Finite Difference Inter-block Hydraulic Conductivity Values for Transient Unsaturated Flow Problems. Water Resources Research, v. 15, n. 1, p. 181-197, 1979.

HILLEL, D. Environmental soil physics. San Diego, California, USA: Academic Press, 1998. $771 \mathrm{p}$.

IWATA, S.; TABUCHI, T.; WARKENTIN, B. P. Soil-Water Interactions: mechanics and applications. Marcel Dekker Inc. New York, NY, USA, 1995. $440 \mathrm{p}$.

KÄMPF, M.; MONTENEGRO, H. On the performance of capillary barriers as landfill cover. Hydrology and Earth System Sciences, v. 4, p. 925-929. 1997.

MIYAZAKI, T. Water flow in soils. Marcel Dekker Inc. New York, New York, USA, 1993. $312 \mathrm{p}$.

MORRIS, C. E.; STORMONT, J. C. Capillary barriers and subtitle d covers: estimating equivalency. Journal of Environmental Engineering, v. 123, n. 1, p. 3-10. 1997.

MUALEM, Y. A new model for predicting the hydraulic conductivity of unsaturated porous media. Water Resources Research, v. 12, n. 3, p. 513522, 1976.

OLDENBURG, C. M.; PRUESS, K. On Numerical Modeling of Capillary Barriers. Water Resources Research, v. 29, n. 4, p. 1045-1056, 1993.

SILVA, P. A. D.; VON SPERLING, E.; SOUZA, L. F. Simulação Numérica do Efeito de Barreira Capilar. Revista Brasileira de Recursos Hídricos, v. 15, n. 3, p. 119-130. 2010.

VAN GENUCHTEN, M. TH. A Closed-form equation for prediction the hydraulic conductivity of unsaturated soils. Soil Science Society America Journal, v. 44, p. 892-898, 1980.

WHISLER, F. D.; KLUTE, A. The Numerical Analysis of Infiltration, Considering Hysteresis, into a Vertical Soil Column at Equilibrium under Gravity. Soil Science Society America Journal, v. 29, p. 489-494. 1965.

YEH, T-C. J.; HARVEY, D. J. Effective Unsaturated Hydraulic of Layered Sands. Water Resources Research, v. 26, n. 6, p. 1271-1279, 1990.

YOUNG, M. H. KARAGUNDUZ, A. ŠIMŮNEK, J.; PENNELL, K. D. A modified upward infiltration method for characterizing soil hydraulic properties. Soil Science Society America Journal, v. 66, p. 57-64. 2002.

ZASLAVSKY, D. Theory of Unsaturated Flow Into a Non-uniform Soil Profile. Soil Science, v. 97, p. 400-410, 1964. 